\title{
Evaluating the role for renal biopsy in T1 and T2 renal masses: A single-centre study
}

Dylan Hoare, $\mathrm{MD}^{1}$; Howard Evans, $\mathrm{MD}^{1}$; Heidi Richards ${ }^{2}$; Rahim Samji, $\mathrm{MD}^{2}$

${ }^{1}$ Division of Urology; ${ }^{2}$ Radiology and Diagnostic Imaging; University of Alberta, Edmonton, AB, Canada

Cite as: Can Urol Assoc J 2018 Feb. 6; Epub ahead of print. http://dx.doi.org/10.5489/cuaj.4831

Published online February 6, 2018

$* * *$

\section{Abstract}

Introduction: Once used primarily in the identification of renal metastasis and lymphomas, various urological bodies are now adopting an expanded role for the renal biopsy. We sought to evaluate the role of the renal biopsy in a Canadian context, focusing on associated adverse events, radiographic burden, and diagnostic accuracy.

Methods: This retrospective review incorporated all patients undergoing ultrasound (US)/computed tomography (CT)-guided biopsies for T1 and T2 renal masses. There were no age or lesion size limitations. The primary outcome of interest was the correlation between initial biopsy and final surgical pathology. A binomial logistic regression analysis was conducted to determine any confounding factors. Secondary outcomes included the accuracy of tumour cell typing, grading, the safety profile and radiographic burden associated with these patients. Results: 148 patients satisfied inclusion criteria for this study. Mean age and lesions size at detection were 60.9 years $( \pm 12.4)$ and $3.6 \mathrm{~cm}( \pm 2.0)$, respectively. Most renal masses were identified with US (52.7\%) or CT (44.6\%). Three patients (2.0\%) experienced adverse events of note. Eighty-six patients (58.1\%) proceeded to radical/partial nephrectomy. Our biopsies held a diagnostic accuracy of $90.7 \%$ (sensitivity $96.2 \%$, specificity $87.5 \%$, positive predictive value 98.7\%, negative predictive value 70.0\%, kappa 0.752, $\mathrm{p}<0.0005$ ). Binomial logistic regression revealed that age, lesion size, number of radiographic tests, time to biopsy, and modality of biopsy (US/CT) had no influence on the diagnostic accuracy of biopsies.

Conclusions: Renal biopsies are safe, feasible, and diagnostic. Their role should be expanded in the routine evaluation of $\mathrm{T} 1$ and $\mathrm{T} 2$ renal masses. 


\section{Introduction}

Given the continued high utilization of cross-sectional imaging, the majority of renal cell carcinomas (RCC) are now detected incidentally. ${ }^{1,2}$ Unlike most malignancies, intervention for suspected kidney cancer often proceeds based on radiographic findings, foregoing tissue diagnosis. ${ }^{3}$ Given the high proportion of clinical T1 and T2 renal lesions comprising this cohort, nephron-sparing approaches currently represent the gold-standard of treatment for many suspected renal cell carcinomas. Due to the associated surgical complications, there has been a recent drive to avoid surgery altogether through ablative techniques. ${ }^{4}$ When factoring in the relatively high frequency of benign pathology found on surgical resection and the desire for noninvasive treatment options, the urologic community has been increasingly motivated to preoperatively risk stratify and diagnose patients with small renal masses.,

Once used primarily in the identification of renal metastasis, lymphomas and abscesses, various urologic bodies are now adopting an expanded role for the renal biopsy. ${ }^{7-9}$ A recent metaanalysis published in European Urology highlighted this increasing acceptance, noting a superb accuracy and a low rate of complications. ${ }^{10}$ We sought to evaluate the role of the renal biopsy in a Canadian academic context, focusing on associated adverse events, radiographic burden, and most importantly, the diagnostic accuracy of this modality.

\section{Methods}

This retrospective review incorporated all patients undergoing biopsies for T1 and T2 renal masses. There were no age or lesion size limitations. Both CT- and ultrasound-guided biopsies were permitted. Patients were excluded if the primary indication for their biopsy was the investigation of medical renal disease or renal cyst aspiration.

Our centre does not employ any standard biopsy request protocol. Prior to undergoing a biopsy, patients will be discussed at length within our combined urology-radiology rounds. Biopsies are performed primarily by body-trained radiologists, and infrequently, by interventional radiology. Ultrasound-guided biopsies employ 18-gauge core needle biopsies, without the use of a coaxial sheath. CT-guided biopsies utilize a 16-gauge coaxial sheath. Radiologists will take between 2 and 4 core samples at their own discretion utilizing the Bard Mission Max-Core, the Cook Quick-Core or the Argon Full Core devices.

Patients were identified from a billings database of renal biopsies maintained by our centre's diagnostic imaging and interventional radiology department. Patient accruement occurred from July 2013 through December 2016 at the Royal Alexandra Hospital in Edmonton, Alberta. Patient demographics were used to identify individuals within our provincial health care repository. Modality and date of initial detection was documented, as was the number of followup images required. Lesion size and radiographically presumed diagnosis were noted as well. Biopsy status included whether the lesion was malignant or benign, its pathologic subtype and Fuhrman grade. This data was paired with, when available, surgical date and pathology to elucidate our outcomes of interest. Surgical status was recorded up to May 2017. 
The primary outcome of interest was the correlation between initial biopsy and final surgical pathology. This diagnostic accuracy was defined as the sum of true positives and true negatives divided by the total number of patients undergoing biopsy. Analysis of sensitivity was conducted with $95 \%$ confidence intervals. A binomial logistic regression analysis was conducted to determine any confounding factors affecting the binary success (diagnostic/non-diagnostic) of renal biopsy.

Secondary outcomes included cell type and Fuhrman grade correlation with final pathology and the safety profile of the intervention as measured by the Clavien-Dindo classification system. ${ }^{11}$ In addition, the radiologic burden of following patients leading up to their biopsy was evaluated. To do so, we quantified the number of surveillance tests (US, CT, MRI, PET, renal scan) patients were exposed to between initial detection and the time of biopsy. All statistical calculations were completed within SPSS.

\section{Results}

148 patients satisfied inclusion criteria for this study, with a higher proportion of males undergoing biopsies (Table 1). Mean age at the time of initial detection was 60.9 years $( \pm 12.4)$. Lesion size at detection had a mean and median size of $3.6 \mathrm{~cm}$ and $3.1 \mathrm{~cm}( \pm 2.0$, range 1.0$15 \mathrm{~cm}$ ) respectively. Most renal masses were identified with US (52.7\%) or CT (44.6\%). On average, patients underwent 2 additional scans prior to their biopsy, with CT representing the most common pre-biopsy modality (Table 2). Imaging tests were conducted for a variety of reasons including improved resolution of the mass, evaluation of interval growth of the lesion and investigation of potential metastatic disease. There was no defined imaging protocol and reasoning for tests was inconsistently reported. As such we were unable to elucidate predictors of increased utilization of diagnostic imaging.

Initial biopsy was conducted within one year of detection for the majority of patients (Table 3). Most were conducted with ultrasound guidance (77.7\%). A small number of patients (11) required repeat biopsy based on suspicious radiographic findings or non-diagnostic results. A greater proportion of patients (41.7\%) required CT guidance for their repeat procedure. Three patients (2.0\%) experienced adverse events of note. Grade I Clavien-Dindo adverse events were not routinely reported and could not be adequately assessed. One patient experienced a small, asymptomatic pneumothorax post-biopsy and another developed a moderate perinephric hematoma associated with pain, both necessitating a short stay in hospital for observation. The final significant adverse event was a grade IVb post-biopsy bleed requiring emergent nephrectomy, inotropic support, and ICU admission. This occurred in a patient with a history of both significant retroperitoneal bleeds and hypercoagulability. Maintained on subcutaneous lowmolecular weight heparin, this was held pre-operatively as is routine in our centre. Pre-biopsy markers of coagulation were normal. Although this was a CT-guided biopsy, it was noted to be exceedingly challenging, requiring traversal of the diaphragm to access the posterior mass, which was also found to be abutting the renal vein. No biopsy-tract seeding was reported. 
Initial biopsy reports found 32 benign (21.6\%), 99 malignant (66.9\%), and 17 nondiagnostic (11.5\%) specimens (Table 4). Eleven patients underwent a second biopsy, one of whom proceeded to a third. Of these repeat biopsies, 4 patients were upgraded from a benign to malignant status, in addition to the 3 patients upgraded from non-diagnostic to malignant. The pathologic subtypes of each biopsy are provided (Table 5).

Eighty-six patients (58.1\%) had a combination of radiographic and/or biopsy results warranting radical/partial nephrectomy, and were suitable operative candidates. Sixty-six (76.7\%) had final surgical pathology correspond directly with their most recent biopsy results (Table 6). Another 9 patients (10.5\%) were deemed malignant on both biopsy and surgical pathology, but had discordant cell types. No patients deemed benign were found to have malignant surgical resections. One patient with a chromophobe subtype on biopsy proceeded to be reclassified on surgical resection as a benign oncocytoma. Of the three patients with both benign biopsy and surgical pathology, two patients proceeded to surgery due to ongoing concerns regarding follow-up and anxiety of their angiomyolipoma. The remaining patient had persistently concerning radiographic features. On final pathology, four (4.7\%) and three (3.5\%) non-diagnostic biopsies were returned benign and malignant, respectively. Our results culminated in a diagnostic accuracy of $90.7 \%$ of patients. A calculated sensitivity of $96.2 \%$ and a positive predictive value of $98.7 \%$ for biopsy detection of malignancy was generated (specificity 87.5\%, negative predictive value 70.0\%, kappa 0.752, $\mathrm{p}<0.0005$ ). Binomial logistic regression revealed that age, lesion size, number of radiographic tests, time to biopsy and modality of biopsy (US/CT) had no influence on the diagnostic accuracy of biopsies (Table 7). In patients who had Fuhrman grade reported on both biopsy and surgical pathology, 22 patients were adequately assessed, 2 underwent down-grading, 17 were upgraded.

\section{Discussion}

Our single-centre retrospective review fits into the growing body of evidence supporting the regular use, safety and high diagnostic accuracy of renal biopsies. A recent meta-analysis from Lorenzo et al presented a diagnostic accuracy for malignancy of $92 \% .{ }^{10}$ Our results compare favourably at $90.7 \%$. Of note, our centre's biopsies are performed primarily by body-trained radiologists under ultrasound guidance, and not by interventional radiology. This highlights a growing comfort with this sampling modality, necessary for its widespread adoption.

Despite the increasing acceptance from radiologists in our centre, and across Canada, there remains some concern from the urologic community regarding the regular use of renal biopsy. As such, routine use of biopsy has yet to become the standard of care in Canada as per the most recent CUA guidelines for the management of the small renal mass. ${ }^{12}$ Similar stances are held by NCCN and the EAU in that renal biopsy remains a complementary, but unnecessary component of the small renal mass workup. ${ }^{13}$ Other more contemporary opinions hold that biopsies should be used to define lesions of likely benign character, or to prepare for ablative/active surveillance strategies. ${ }^{14}$ The American Society of Clinical Oncology recently proclaimed that when accounting for competing mortality risks and tumour-specific findings, all 
small renal masses should undergo biopsy if management has the potential to be altered. ${ }^{15}$ With the advent of novel biomarkers and a greater appreciation of immunohistochemistry, tissue diagnosis will be of even greater importance. ${ }^{14}$

Non-diagnostic results remain one of the most oft-cited concerns with kidney biopsies. Our series possessed an initial non-diagnostic rate of $11.5 \%$. When enabling the use of repeat biopsies, this number decreased to $9.5 \%$. A number of these samples were reported as chromophobe vs oncocytoma, a well documented diagnostic dilemma. ${ }^{16}$ This non-diagnostic rate ultimately compares well with other Canadian series, and highlights the importance of being open to repeat sampling. ${ }^{17}$ Importantly, a non-diagnostic status should not preclude surgery. In our series, $50 \%$ of non-diagnostic cases proceeded to nephrectomy and/or repeat biopsy. Given that our non-diagnostic rate represents an improvement over the literature reported of benign nephrectomy, this indicates a clinical advantage to the use of core needle sampling, despite the occasional diagnostic uncertainty. Identification of specific cell types remains a strong, albeit, imperfect feature of biopsies. ${ }^{18}$ This serves as an important feature, particularly in the comorbid patient where prognosticating is a critical aspect of their care. Fuhrman grade characterization remains highly variable however, both in our series and throughout the literature. ${ }^{19}$ This is believed to be in large part due to the grade heterogeneity observed in renal masses. ${ }^{20}$ The concern regarding adverse events has been dampened with experience and evidence supporting low complication rates throughout the literature. ${ }^{21}$ Our review was comprised of only one event requiring operative management and two additional cases necessitating 24-hour monitoring. The Clavien IVb event we experienced highlights the importance of patient selection, as the patient had a known bleeding diathesis and may have benefitted from active surveillance. Based on current ASCO guidelines, this patient would have met the relative indications for active surveillance as well. ${ }^{15}$ It remains to be seen what role the renal biopsy will have in future active surveillance regimens. ${ }^{13}$ No needle-tract seeding was observed in our review. Outside of rare reports, this remains consistent with the current body of evidence. ${ }^{22,23}$ In addressing the limitations of our study, we identify that this is in fact a retrospective series. The evaluation of renal biopsy will require prospectively randomized data before definitive guidelines can be established. ${ }^{10}$ In addition, our sample requires long-term follow-up to strengthen our outcomes of interest. The theoretical risk of needle tract seeding or deterioration in renal function may take years to develop. In addition, the assumption was made that benign biopsies not proceeding to surgery were definitively non-malignant. It is possible that in the years to come, these masses could begin demonstrating malignant character and require repeat biopsy or surgical resection.

Moving forward, our centre would like to analyze the long-term follow-up of these patients. We plan to revisit our cohort in five years to assess rates of recurrence and malignant transformation. This will provide useful insight into the true negative rate or specificity of the renal biopsy. In addition, we would like to perform a cost-benefit analysis. The goal is that a renal biopsy will help eliminate the unnecessary cost of an operation planned for a benign lesion. 
The competing factors are the cost of the biopsy and the plethora of radiographic tests that are often ordered in surveillance regimens. Born out of a likely lack of trust in renal biopsy results, our study demonstrated a high radiographic burden attached to these patients. This undoubtedly factors into the cost analysis, but may improve with time as urologists and radiologists alike grow more comfortable with this test.

\section{Conclusion}

Renal biopsies are safe, feasible and diagnostic. Their role should be expanded in the routine evaluation of $\mathrm{T} 1$ and $\mathrm{T} 2$ renal masses. 


\section{References}

1. Volpe A, Panzarella T, Rendon RA et al. The natural history of incidentally detected small renal masses. Cancer 2004;100:738-45.

2. Rossi SH, Hsu R, Blick C et al. Meta-analysis of the prevalence of renal cancer detected by abdominal ultrasonography. Br J Surg 2017;104:658-59.

3. Kockelbergh R, Griffiths L. Renal Tumour Biopsy-A New Standard of Care? Eur Urol 2016;69:674-5.

4. Volpe A, Finelli A, Gill IS et al. Rationale for percutaneous biopsy and histologic characterisation of renal tumours. Eur Urol 2012;62:491-504.

5. Pahernik S, Zielger S, Roos F et al. Small renal tumors: correlation of clinical and pathological features with tumor size. J Urol 2007;178:414-7.

6. Remzi M, Ozsoy M, Klingler $\mathrm{H}$ et al. Are small renal tumors harmless? Analysis of histopathological features according to tumors $4 \mathrm{~cm}$ or less in diameter. $J$ Urol 2006;176:896-9.

7. Novick AC, Campbell SC, Belldegrun A et al. Guideline for management of the clinical stage 1 renal mass. J Urol 2009;182:1271-9.

8. Richard PO, Jewett MA, Bhatt JR et al. Renal tumor biopsy for small renal masses: a single-center 13-year experience. Eur Urol 2015;68:1007-13.

9. Halverson SJ, Kunju LP, Bhalla R et al. Accuracy of determining small renal mass management with risk stratified biopsies: confirmation by final pathology. J Urol 2013;189:441-6.

10. Lorenzo M, Dabestani S, Lam TB et al. Systematic review and meta-analysis of diagnostic accuracy of percutaneous renal tumour biopsy. Eur Urol 2016;69:660-73.

11. Dindo D, Demartines N, Clavien P. Classification of surgical complications: a new proposal with evaluation in a cohort of 6336 patients and results of a survey. Ann Surg 2004;240:205-13.

12. Jewett MA, Rendon R, Lacombe $L$ et al. Canadian guidelines for the management of small renal masses (SRM). Can Urol Assoc J 2015;9:160-3.

13. Ljungberg B, Bensalah K, Canfield S et al. EAU guidelines on renal cell carcinoma: 2014 update. Eur Urol 2015;67:913-24. 2017 update Epub.

14. Burruni R, Lhermitte B, Cerantola Y et al. The role of renal biopsy in small renal masses. Can Urol Assoc J 2016;10:E28-E33.

15. Finelli A, Ismaila N, Bro B et al. Management of small renal masses: American Society of Clinical Oncology clinical practice guideline. J Clin Oncol 2017;35:668-80.

16. Chang S, Loda M. Pathology and Molecular Pathology of Renal Cancer. Pathology and Epidemiology of Cancer 2017:335-46. 
17. Leveridge MJ, Finelli A, Kachura J et al. Outcomes of small renal mass needle core biopsy, nondiagnostic percutaneous biopsy, and the role of repeat biopsy. Eur Urol 2011;60:578-84.

18. Veltri A, Garetto I, Tosetti I et al. Diagnostic accuracy and clinical impact of imagingguided needle biopsy of renal masses. Retrospective analysis on 150 cases. Eur Radiol 2011;21:393-401.

19. Abel EJ, Culp SH, Matin SF et al. Percutaneous biopsy of primary tumour in metastatic renal cell carcinoma to predict high risk pathological features: comparison with nephrectomy assessment. J Urol 2010;184:1877-81.

20. Ball MW, Bezerra SM, Gorin MA et al. Grade heterogeneity in small renal masses: Potential implications for renal mass biopsy. J Urol 2015;193:36-40.

21. Volpe A, Kachura JR, Geddie WR et al. Techniques, safety and accuracy of sampling of renal tumors by fine needle aspiration and core biopsy. J Urol 2007;178:379-86.

22. Chang DTS, Sur H, Lozinskiy M et al. Needle tract seeding following percutaneous biopsy of renal cell carcinoma. Korean J Urol 2015;56:666-9.

23. Andersen, MFB, Norus TP. Tumor seeding with renal cell carcinoma after renal biopsy. Urol Case Rep 2016;9:43-4. 
Figures and Tables

\begin{tabular}{|c|c|c|c|c|}
\hline & Male & Females & Total & \\
\hline \multirow[t]{2}{*}{ Sample } & 91 (61.5\%) & 57 (38.5\%) & 148 & \\
\hline & US & $\mathrm{CT}$ & MRI & PET \\
\hline \multirow[t]{2}{*}{$\begin{array}{l}\text { Means of } \\
\text { detection }\end{array}$} & $78(52.7 \%)$ & 66 (44.6\%) & $3(2.0 \%)$ & $1(0.7 \%)$ \\
\hline & Median & Mean & SD & \\
\hline Age at detection & 61.4 years & 60.9 years & \pm 12.4 & \\
\hline Size at detection & $3.1 \mathrm{~cm}$ & $3.6 \mathrm{~cm}$ & \pm 2.0 & \\
\hline
\end{tabular}

CT: computed tomography; MRI: magnetic resonance imaging; SD: standard deviation; US: ultrasound.

\begin{tabular}{|l|c|c|c|c|c|}
\hline \multicolumn{4}{|l|}{ Table 2. Followup imaging } & & \\
\hline \multicolumn{1}{|c|}{ Modality } & Median & Mean & SD & $\begin{array}{c}\text { Patients with only 1 } \\
\text { followup test }\end{array}$ & $\begin{array}{c}\text { Patients with } \geq \mathbf{2} \\
\text { followup tests }\end{array}$ \\
\hline US & 0 & 0.9 & \pm 1.6 & 23 & 36 \\
\hline CT & 1 & 1.3 & \pm 1.3 & 82 & 36 \\
\hline MRI & 0 & 0.3 & \pm 0.6 & 27 & 10 \\
\hline Pet & 0 & 0.05 & \pm 0.2 & 27 & 1 \\
\hline Bone scan & 0 & 0.06 & \pm 0.3 & 7 & 1 \\
\hline Renal scan & 0 & 0.02 & \pm 0.1 & 3 & 82 \\
\hline Total scans & 2 & 2.6 & \pm 2.8 & 50 & \\
\hline
\end{tabular}

CT: computed tomography; MRI: magnetic resonance imaging; SD: standard deviation; US: ultrasound.

\begin{tabular}{|c|c|c|c|c|c|c|c|c|c|}
\hline & \multirow[t]{2}{*}{$\mathbf{n}$} & \multicolumn{3}{|c|}{ Age } & \multicolumn{3}{|c|}{$\begin{array}{c}\text { Time from detection to } \\
\text { biopsy (months) }\end{array}$} & \multicolumn{2}{|c|}{ Modality } \\
\hline & & Median & Mean & SD & Median & Mean & SD & US-guided & CT-guided \\
\hline Biopsy 1 & 148 & 62.1 & 61.8 & \pm 12.6 & 3.7 & 11.0 & \pm 17.7 & $115(77.7 \%)$ & $33(22.3 \%)$ \\
\hline Biopsy 2 & 11 & 53.7 & 53.3 & \pm 13.8 & 4.9 & 11.2 & \pm 9.0 & $6(54.5 \%)$ & $5(45.5 \%)$ \\
\hline Biopsy 3 & 1 & 55.3 & 55.3 & $\mathrm{~N} / \mathrm{a}$ & 23.8 & 23.8 & N/a & $1(100 \%)$ & 0 \\
\hline
\end{tabular}

CT: computed tomography; SD: standard deviation; US: ultrasound. 


\begin{tabular}{|l|c|c|c|c|}
\hline Table 4. Biopsy status & \multicolumn{3}{|c|}{ Status } \\
\hline & n & \multicolumn{3}{|c|}{} \\
\hline & & Benign & Malignant & Non-diagnostic \\
\hline Biopsy 1 & 148 & $32(21.6 \%)$ & $99(66.9 \%)$ & $17(11.5 \%)$ \\
\hline Biopsy 2 & 11 & $3(27.3 \%)$ & $8(72.7 \%)$ & 0 \\
\hline Biopsy 3 & 1 & $1(100 \%)$ & 0 & 0 \\
\hline At final biopsy & 148 & $28(18.9 \%)$ & $106(71.6 \%)$ & $14(9.5 \%)$ \\
\hline
\end{tabular}

\begin{tabular}{|c|c|c|}
\hline Table 5. Biopsy pathology & & \\
\hline & Initial biopsy pathology & Final biopsy pathology \\
\hline Total malignant & $99(66.9 \%)$ & $106(71.6 \%)$ \\
\hline Clear cell & $55(37.2 \%)$ & $60(40.5 \%)$ \\
\hline Papillary & $20(13.5 \%)$ & $20(13.5 \%)$ \\
\hline Type 1 & $10(6.8 \%)$ & $10(6.8 \%)$ \\
\hline Type 2 & $4(2.7 \%)$ & $4(2.7 \%)$ \\
\hline Undefined/other & $6(4.1 \%)$ & $6(4.1 \%)$ \\
\hline Chromophobe & $9(6.1 \%)$ & $10(6.8 \%)$ \\
\hline Epithelioid angiomyolipoma & $1(0.7 \%)$ & $1(0.7 \%)$ \\
\hline Lymphoma & $5(3.4 \%)$ & $5(3.4 \%)$ \\
\hline Urothelial & $3(2.0 \%)$ & $3(2.0 \%)$ \\
\hline Sarcomatoid & $1(0.7 \%)$ & $1(0.7 \%)$ \\
\hline Metastatic from other site & $1(0.7 \%)$ & $1(0.7 \%)$ \\
\hline Undifferentiated malignant & $4(2.7 \%)$ & $5(3.4 \%)$ \\
\hline Total benign & $32(21.6 \%)$ & $28(18.9 \%)$ \\
\hline Oncocytoma & $12(8.1 \%)$ & $11(7.4 \%)$ \\
\hline Angiomylipoma & $6(4.1 \%)$ & $6(4.1 \%)$ \\
\hline Arteriosclerosis/glomerulosclerosis & $3(2.0 \%)$ & $2(1.4 \%)$ \\
\hline Hematoma & $2(1.4 \%)$ & $2(1.4 \%)$ \\
\hline Necrotizing granulomatous reaction & $1(0.7 \%)$ & $1(0.7 \%)$ \\
\hline No abnormal histology & $8(5.4 \%)$ & $6(4.1 \%)$ \\
\hline Total non-diagnostic & $17(11.5 \%)$ & $14(9.5 \%)$ \\
\hline $\begin{array}{l}\text { Non-diagnostic chromophobe vs. } \\
\text { oncocytoma }\end{array}$ & $5(3.4 \%)$ & $5(3.4 \%)$ \\
\hline Other non-diagnostic & $12(8.1 \%)$ & $9(6.1 \%)$ \\
\hline
\end{tabular}




\begin{tabular}{|c|c|c|c|}
\hline \multirow[t]{2}{*}{ Biopsy status } & \multicolumn{2}{|c|}{$\begin{array}{l}\text { Surgical status } \\
\end{array}$} & \multirow[b]{2}{*}{ Total } \\
\hline & Malignant & Benign & \\
\hline $\begin{array}{l}\text { Malignant correct cell } \\
\text { type }\end{array}$ & $66(76.7 \%)$ & 0 & 66 \\
\hline Malignant total & $75(87.2 \%)$ & $1(1.2 \%)$ & 76 \\
\hline Benign & 0 & $3(3.5 \%)$ & 3 \\
\hline Non-diagnostic & $3(3.5 \%)$ & $4(4.7 \%)$ & 7 \\
\hline Total & 78 & 8 & 86 \\
\hline
\end{tabular}

True positives: total malignant biopsy pathology and malignant surgical pathology; false positives: total malignant biopsy pathology and benign surgical pathology; true negatives: benign + non-diagnostic biopsy pathology and benign surgical pathology; False negative = benign + non-diagnostic biopsy pathology and malignant surgical pathology.

\begin{tabular}{|l|c|c|c|c|c|c|}
\hline Table 7. Binomial logistic regression of variables affecting diagnostic accuracy of biopsies \\
\hline Covariate & $\mathbf{B}$ & Bias & Std. error & $\mathbf{p}$ & \multicolumn{2}{|c|}{ 95\% confidence interval } \\
\hline & & & & & Lower & Upper \\
\hline Age & -0.019 & -0.158 & 6.352 & $\mathbf{0 . 5 6 7}$ & -0.131 & 0.115 \\
\hline Lesion size & 0.516 & 2.331 & 38.569 & $\mathbf{0 . 1 9 1}$ & -0.064 & 3.209 \\
\hline $\begin{array}{l}\text { Total radiographic } \\
\text { tests }\end{array}$ & 0.092 & 0.155 & 16.463 & $\mathbf{0 . 6 3 9}$ & -0.550 & 1.342 \\
\hline Time to biopsy & -0.269 & 0.264 & 34.160 & $\mathbf{0 . 4 5 0}$ & -2.974 & 2.809 \\
\hline Modality (US/CT) & -1.054 & -2.815 & 48.432 & $\mathbf{0 . 2 2 7}$ & -5.115 & 18.338 \\
\hline
\end{tabular}

CT: computed tomography; SD: standard deviation; US: ultrasound. 